\title{
A COMPARISON BETWEEN DUCTED TURBINE THEORY AND INVISCID SIMULATION
}

\author{
S. McLaren-Gow*, P. Jamieson ${ }^{\dagger}$, J.M.R. Graham \\ *University of Strathclyde, United Kingdom, scott.mclaren-gow@strath.ac.uk \\ ${ }^{\dagger}$ University of Strathclyde, United Kingdom, peter.jamieson@strath.ac.uk \\ *Imperial College London, United Kingdom, m.graham@imperial.ac.uk
}

Keywords: ducted wind turbines, diffuser augmentation, aerodynamics, panel methods

\begin{abstract}
This paper describes the use of an inviscid approach to model a ducted turbine and a comparison of simulation results with a particular one-dimensional theory. The aim of the investigation was to gain a better understanding of the ideal diffuser, a concept that is developed in the theory. An optimisation of duct shape and a set of simulations with a varied group of shapes showed that duct length, inlet radius and outlet radius are not sufficient to define an ideal diffuser. A set of simulations with cylindrical ducts confirmed the theory that a duct optimised for one loading is less than ideal at other loadings.
\end{abstract}

\section{Introduction}

Appropriate ducting around the circumference of a wind turbine rotor can augment the mass flow through that rotor and so increase the power coefficient achieved. Research on these ducted turbines - some also known as Diffuser Augmented Wind Turbines - has a long history, with many authors attempting to develop the concept and improve wind turbine performance. Notable contributions include early work by Lilley and Rainbird [1], wide ranging investigations by Foreman et al [2-4] and Igra [5] and the attempted development of a full scale commercial turbine by Vortec Energy [6]. Research has continued since and several small scale ducted turbines have entered production [7].

These investigations have generally taken one of three approaches to modelling a ducted turbine: one-dimensional analysis, experimentation with models in a wind tunnel and simulations with Computational Fluid Dynamics (CFD). While one-dimensional analyses can provide a more general understanding of the principles of ducted turbine performance, empirical parameters are invariably required to provide results for a particular duct. Experiments and CFD simulations do not suffer this limitation, but with the downside of much increased complexity.

This work concerns an alternative approach in which the duct and wake are modelled using a panel method. This does not require empirical parameters and is less complex than the experiment and CFD approaches. The main advantage, however, is the inviscid nature of the method. This allows the fundamental aspects of ducted turbine performance to be examined separate from the limitations imposed by viscosity. Importantly, an inviscid approach also allows for the validation of one-dimensional theory without concern for differences caused by viscous effects.

Of particular interest is the one-dimensional theory developed by Jamieson [8]. This introduces the concept of an ideal diffuser: one of infinitely variable shape to account for the changing interaction between the diffuser and a variably loaded rotor. While this concept is obviously entirely theoretical in nature, it has definite consequences for the design of real ducted turbines. Where the ideal diffuser is optimised for every rotor loading by virtue of its variable geometry, the real diffuser of fixed geometry can be optimum for only one loading. Further, each ideal diffuser has a corresponding set of real diffusers, each potentially optimised for one loading. If this concept was fully understood and the theory validated, it would provide the opportunity to maximise the performance of a real ducted turbine. In turn, this could lead to a reduced cost of wind energy.

The exact specifications that define an ideal diffuser and the associated set of real diffusers have not yet been found. This paper will discuss results which test one possibility: that an ideal diffuser is defined by duct length, inlet radius and outlet radius. Continuing the investigation of ideal and optimum diffusers, the thesis that a duct optimum at one loading will be less than optimum at other loadings is then evaluated.

\section{Modelling Approach}

A previously developed axisymmetric vortex panel method was employed to model the duct and turbine wake [9]. A more complete description of the solution process can be found in the previous work. Each duct and turbine wake panel consisted, in general, of a discrete vortex ring and a collocation point at which boundary conditions were enforced. The rotor itself was represented simply as an actuator disc generating a pressure drop that was applied uniformly across the duct cross-section.

The duct was modelled without thickness and was formed by a set of vortex rings unevenly distributed along its length. The 
half cosine method was applied to the length along the duct surface, rather than to axial position, to achieve this Collocation points were placed at half the distance along the duct surface between each ring, with one collocation point a half panel length downstream of the last ring. When solving, the duct vortex ring strengths were calculated to achieve zero normal flow on the duct boundary.

A constant panel length was used to define the wake, with the vortex rings and collocation points placed at the centre of each panel. The panel length was set equal to the length along the duct surface between the last two duct collocation points to prevent numerical problems caused by an abrupt change in panel length. The discrete wake elements were terminated downstream by a semi-infinite vortex cylinder [10] in order to improve accuracy and avoid the unrealistic end shape of a finite length wake. When solving, the wake vortex strengths were calculated so to prevent a pressure discontinuity across the wake.

The solution process was iterative in nature. First, duct and wake ring strengths were solved in an inner layer of iteration. Then, a new wake shape was calculated so that the wake panels remained on the wake boundary and so that the wake could take up a converged shape satisfying the force-free condition. These two steps were repeated until convergence of the shape was reached.

\section{Results and Discussion}

All the results presented here use the standard wind turbine definitions; power and thrust coefficients, $\mathrm{C}_{\mathrm{p}}$ and $\mathrm{C}_{\mathrm{t}}$, are referenced to the rotor area and free stream velocity. The rotor was placed at the duct inlet in all cases.

\subsection{Shape Optimisation}

The theory states that two real ducts associated with one ideal diffuser but optimum at different loadings will be different shapes. This provided one avenue to test the ideal duct definition under consideration: an optimisation algorithm was used to vary duct shape in order to maximise mass flow rate through the rotor, once at $\mathrm{C}_{\mathrm{t}}=0$ and once at $\mathrm{C}_{\mathrm{t}}=8 / 9$.

The radius of the duct was specified at three axial positions, with a complete description of the shape provided by interpolating between these points with a cubic Hermite spline that respected monotonicity. The radius and axial position of the first and last points were fixed. This provided a constant length, inlet radius and outlet radius for the ducts. The optimisation algorithm acted to vary the axial position and radius of the central point, with bounds formed by the axial positions and radii of the end points.

Under these restrictions, the optimisation process indicated that the best shape consisted of a sharp right angle for both loadings, as shown in Figure 1. These results therefore do not support the ideal duct definition being considered. However, certain limitations in the approach mean that the results are not conclusive. Firstly, only a subset of all the possible shapes joining a fixed inlet and outlet can be represented by the three point approach taken. Secondly, even within the constraints specified, the optimisation algorithm may have found a local rather than global optimum.

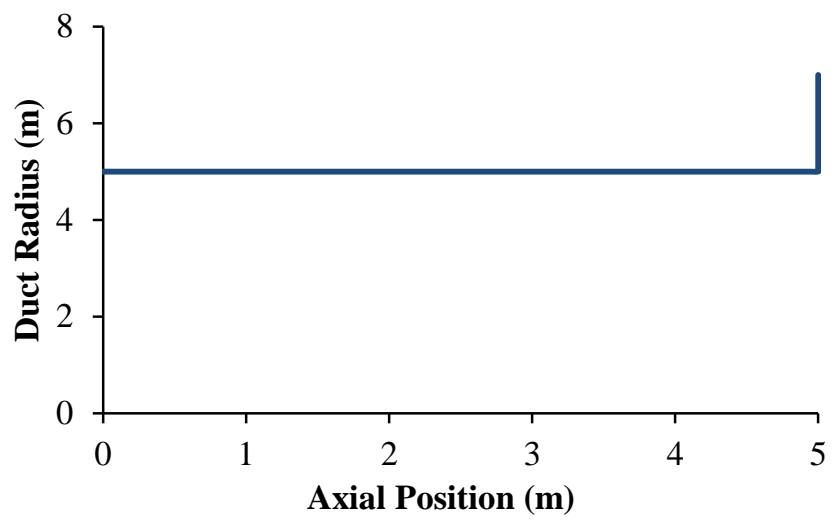

Figure 1: Shape indicated as optimum

Comparison with another aspect of the theory does in fact suggest that the shape found may not be the true optimum, at least for $C_{t}=8 / 9$. This aspect states that the greatest power coefficient in a duct should be achieved at $C_{t}=8 / 9$, otherwise it is not the best performing real version of the associated ideal diffuser. Further simulations showed that the shape found here produced the greatest power output at $\mathrm{C}_{\mathrm{t}}=0.79$, so a more optimum shape may exist.

\subsection{Shape Investigation}

Two further aspects of the theory were used to test the ideal diffuser definition under consideration. First, $C_{\text {topt }}-C_{t}$ for maximum power - is expected to increase with $\mathrm{C}_{\mathrm{pmax}}$ for all ducts associated with one ideal diffuser. Second, the induction in the ideal diffuser unloaded, $\mathrm{a}_{0}$, can be estimated from the real duct results. All real ducts associated with a particular ideal diffuser must provide the same estimate.

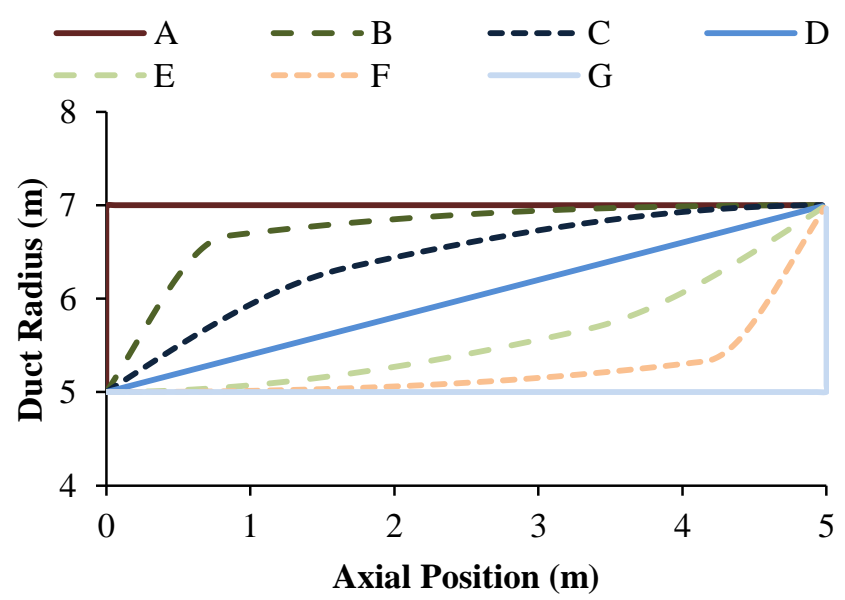

Figure 2: Shapes A-G used in shape investigation.

The shapes for these tests were specified using the same 3 point spline approach used in the shape optimisation 
simulations. The position of the central point was varied from one corner of the bounds to the other to provide the shapes shown in Figure 2. These were not limited to practical designs as the focus was on the theoretical implications of the results.

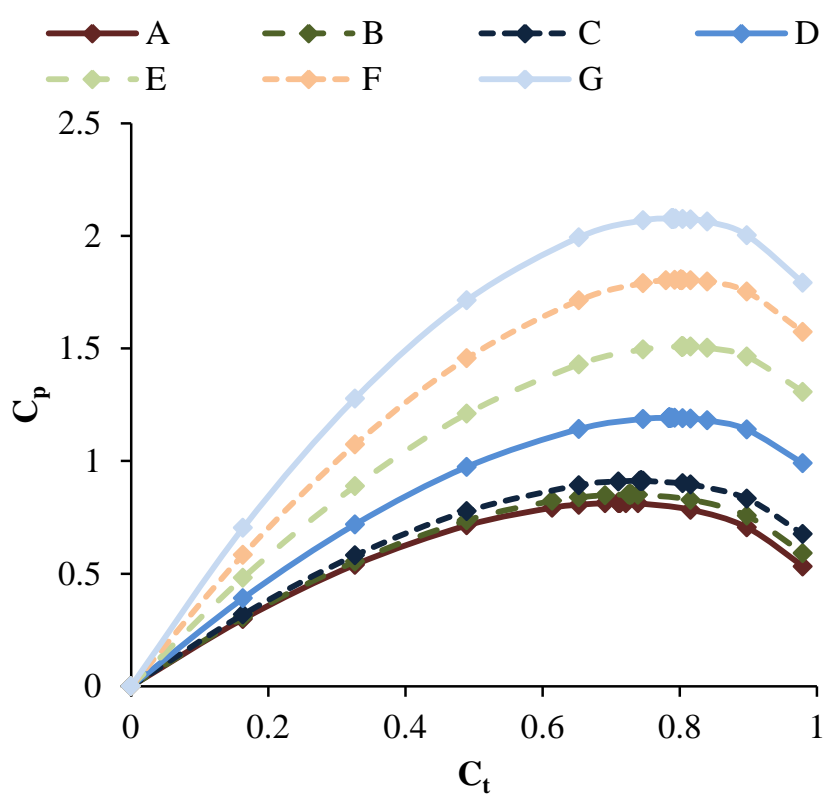

Figure 3: Power and thrust coefficient results for shapes A-G

\begin{tabular}{|c|c|c|c|c|}
\hline Shape & $\eta$ & $\mathrm{a}_{0}$ & $\mathrm{C}_{\mathrm{pmax}}$ Ideal & $\mathrm{C}_{\mathrm{pmax}}$ Achieved \\
\hline $\mathrm{A}$ & 0.80 & -1.23 & 1.32 & 0.81 \\
$\mathrm{~B}$ & 0.82 & -1.26 & 1.34 & 0.85 \\
$\mathrm{C}$ & 0.84 & -1.33 & 1.38 & 0.91 \\
$\mathrm{D}$ & 0.88 & -1.78 & 1.65 & 1.19 \\
$\mathrm{E}$ & 0.90 & -2.39 & 2.01 & 1.51 \\
$\mathrm{~F}$ & 0.90 & -3.16 & 2.46 & 1.81 \\
$\mathrm{G}$ & 0.89 & -4.15 & 3.05 & 2.08 \\
\hline
\end{tabular}

Table 1: Results for shapes A-G and estimates for the associated ideal diffusers.

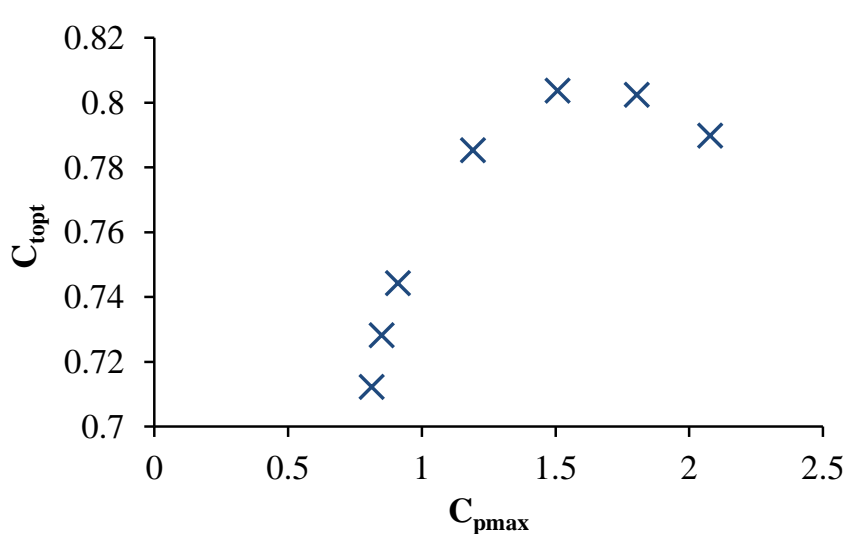

Figure 4: Variation of thrust coefficient for maximum power coefficient, for shapes A-G.

Power and thrust coefficient results are shown in Figure 3. Performance was found to reduce as the shape became further removed from that produced by the optimisation algorithm, as was expected. Figure 4 shows the optimum thrust coefficient and the maximum power coefficient. The optimum loading was found to decrease with increasing $\mathrm{C}_{\mathrm{pmax}}$ after a $\mathrm{C}_{\mathrm{pmax}}$ of around 1.6; this suggests the ducts are not real versions of the same ideal diffuser.

Table 1 shows the diffuser efficiency, $\eta$, - the ratio of $C_{\text {topt }}$ to $8 / 9$ - and maximum power coefficient achieved for each of the ducts. It also shows the estimated $\mathrm{a}_{0}$ value and maximum power coefficient expected from the associated ideal diffuser. These results again provide evidence against the definition of ideal diffuser being considered, as the $\mathrm{a}_{0}$ value is not consistent between ducts.

\subsection{Cylindrical Duct Investigation}

As has been shown, it may be difficult to define the set of real ducts associated with an ideal diffuser. In general it is also difficult to determine $a_{0}$ for a particular ideal diffuser. However, the ideal for $a_{0}=0$ is simply the open flow case as zero induction is established by the absence of a duct.

A set of real ducts optimum for zero loading and corresponding to the ideal diffuser with $\mathrm{a}_{0}=0$ is also easily determined. Since the velocity vectors are all parallel and the flow field is completely uniform when there is no rotor loading, any cylindrical duct with its axis aligned with the flow will induce zero induction - matching the ideal - and so be optimum. In this simple case, the thesis that any ducts that are optimum at one loading will be sub-optimum at other loadings can be tested.

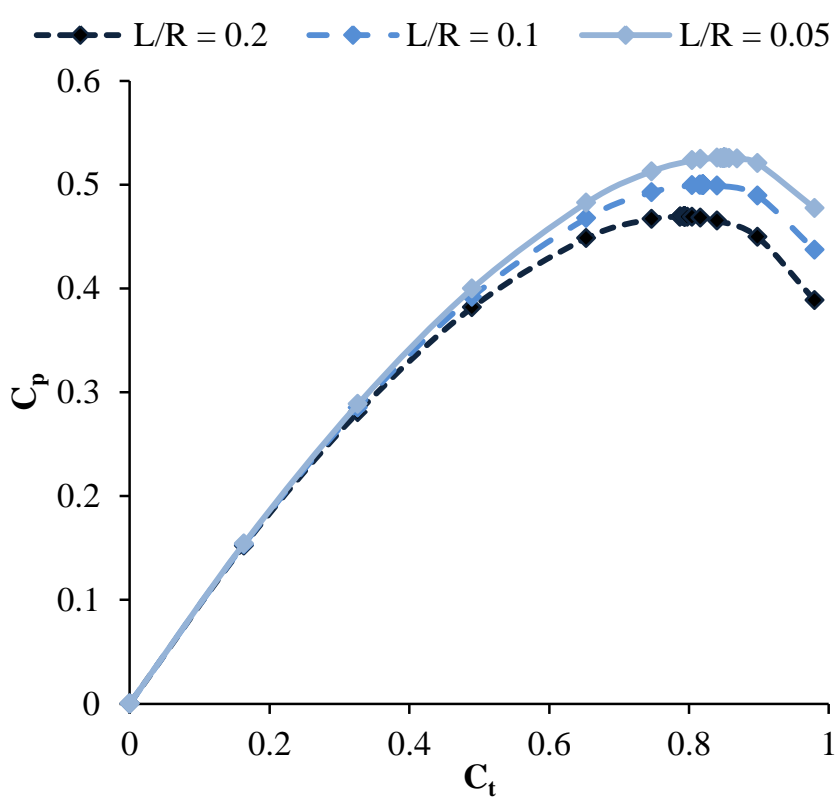

Figure 5: Power and thrust coefficient results for cylindrical ducts with varied length $(\mathrm{L})$ to radius $(\mathrm{R})$ ratios.

Figure 5 shows power and thrust coefficient results for three cylindrical ducts with varying length to duct radius ratios. At all lengths, the power coefficient is lower than the ideal open flow value for loadings other than zero. These results therefore do show the cylindrical ducts to be optimum at only 
one loading. These results also show multiple real ducts that are optimum at the same loading, even though the ducts are associated with the same ideal diffuser. It is unclear if this is possible in general or if this test was a special case.

It can also be seen that increasing length decreases the maximum power coefficient achieved and, as Figure 6 highlights, decreases the optimum thrust coefficient from the ideal value of $8 / 9$. Without a duct, the streamtube passing through the rotor is free to take up the optimum shape for each loading. A cylindrical duct prevents this while also not providing any mass flow augmentation to counteract the less than ideal wake shape. As a shorter duct has less influence on the flow, the results tend towards the ideal values as the duct is shortened.

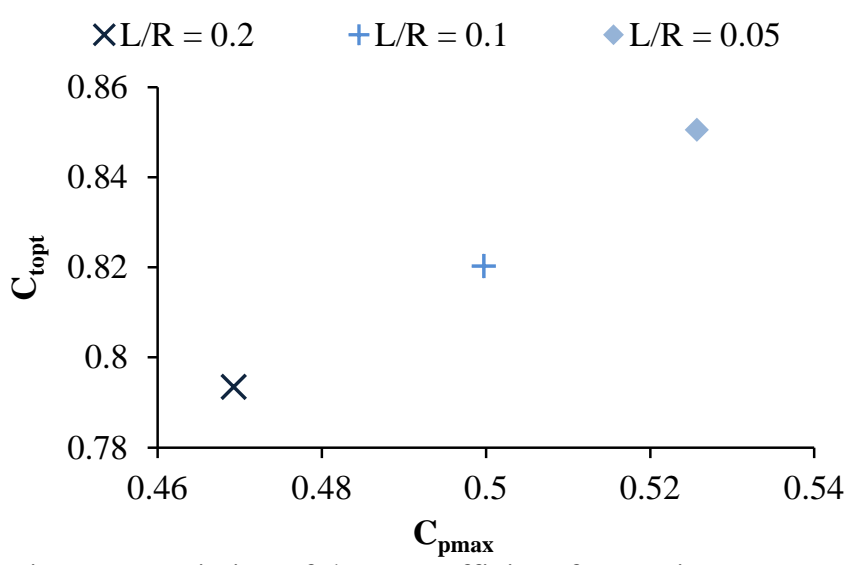

Figure 6: Variation of thrust coefficient for maximum power coefficient for cylindrical ducts with varied length (L) to radius $(\mathrm{R})$ ratios.

\section{Conclusions}

An inviscid simulation method has been used to model the performance of a ducted turbine. An optimisation algorithm was used to find that the same shape produced the greatest mass flow rate at two different loadings, for ducts with the same length, inlet radius and outlet radius. This suggested that these parameters cannot be used to define an ideal diffuser, but limitations in the optimisation approach mean that the result is inconclusive. Further simulations with a range of duct shapes under the same geometric restrictions provided more conclusive evidence: $\mathrm{C}_{\text {topt }}$ did not consistently increase with $\mathrm{C}_{\text {pmax }}$, and a different estimate of $\mathrm{a}_{0}$ was found for each shape. These results are not consistent with a set of ducts associated with the same ideal diffuser.

This work has therefore tested the theory that length, inlet radius and outlet radius fully specify an ideal diffuser and so specify the associated set of real diffusers. This was found not to be the case. Other possibilities exist; for example, multiple ideal diffusers may share the same values for these three parameters and so further specifications may be required to uniquely identify a single ideal diffuser. It is also possible that one or more of these parameters are not fixed: an ideal diffuser may be of variable length as well as shape, for instance.

A final set of simulations investigated one case where a set of real ducts that were optimum unloaded could be associated with an ideal diffuser. The results found were consistent with the theory that a real duct optimum at one loading will give less than ideal performance at other loadings.

\section{Acknowledgements}

This work has been funded by the EPSRC, under project reference number EP/G037728/1.

\section{References}

[7] G. J. W. van Bussel, "The science of making more torque from wind: Diffuser experiments and theory revisited," Journal of Physics: Conference Series, vol. 75, p. 012010, 2007.

[8] P. Jamieson, "Generalized Limits for Energy Extraction in a Linear Constant Velocity Flow Field," Wind Energy, vol. 11, pp. 445-457, 2008.

[9] S. McLaren-Gow, P. Jamieson, and J. M. R. Graham, "An Inviscid Approach to Ducted Turbine Analysis," in European Wind Energy Association Annual Conference, Vienna, 2013.

[10] I. S. Gibson, "On the velocity induced by a semiinfinite vortex cylinder: with the extension to the short solenoid," Aeronautical Journal, vol. 78, pp. 262-268, 1974. 